\title{
On the supersymmetric extension of Gauss-Bonnet like gravity
}

\author{
P.K. Concha, ${ }^{a, b}$ M.C. Ipinza, ${ }^{c, d, e}$ L. Ravera ${ }^{d, e}$ and E.K. Rodríguez ${ }^{a, b}$ \\ ${ }^{a}$ Departamento de Ciencias, Facultad de Artes Liberales, Universidad Adolfo Ibáñez, \\ Av. Padre Hurtado 750, Viña del Mar, Chile \\ ${ }^{b}$ Instituto de Ciencias Físicas y Matemáticas, Universidad Austral de Chile, \\ Casilla 567, Valdivia, Chile \\ ${ }^{c}$ Departamento de Fúsica, Universidad de Concepción, \\ Casilla 160-C, Concepción, Chile \\ ${ }^{d}$ DISAT, Politecnico di Torino, \\ Corso Duca degli Abruzzi 24, I-10129 Torino, Italia \\ ${ }^{e}$ Istituto Nazionale di Fisica Nucleare (INFN), Sezione di Torino, \\ Via Pietro Giuria 1, 10125, Torino, Italia \\ E-mail: patillusion@gmail.com, marcelo.calderon@polito.it, \\ lucrezia.ravera@polito.it, everodriguezd@gmail.com
}

ABSTRACT: We explore the supersymmetry invariance of a supergravity theory in the presence of a non-trivial boundary. The explicit construction of a bulk Lagrangian based on an enlarged superalgebra, known as AdS-Lorentz, is presented. Using a geometric approach we show that the supersymmetric extension of a Gauss-Bonnet like gravity is required in order to restore the supersymmetry invariance of the theory.

KEYwords: Supergravity Models, Gauge Symmetry

ARXiv EPrint: 1607.00373 


\section{Contents}

1 Introduction 1

$2 \quad A d S$-Lorentz supergravity and rheonomy approach $\quad 2$

2.1 Curvatures parametrization 3

2.2 Rheonomic construction of the Lagrangian 4

$\begin{array}{ll}2.3 & \text { Supersymmetry transformation laws }\end{array}$

$\begin{array}{lll}3 & \text { Supersymmetry invariance in the presence of a boundary } & 7\end{array}$

4 Comments and possible developments $\quad 10$

\section{Introduction}

The presence of a boundary in the context of (super)gravity has been studied with great interest these last 40 years. In particular, the inclusion of boundary terms plays an important role for the study of the fruitful duality between string theory on asymptotically AdS space-time and a quantum field theory living on the boundary (AdS/CFT correspondence) [1-4]. The study of bulk and boundary theories has led to the development of the so called holographic renormalization. Indeed, UV divergences in the field theory (boundary) are related to IR divergences on the gravitational side (bulk) which can be dealt through the holographic renormalization procedure [5-7], adding appropriate counterterms to the boundary.

At the bosonic level, the introduction of the topological Gauss-Bonnet term to the four-dimensional AdS gravity allows to regularize the action and the related conserved charges [8-13]. Remarkably, the inclusion of the Gauss-Bonnet term does not require to impose Dirichlet boundary conditions on the fields. On the other hand, the addition of boundary terms to supergravity has been considered in different approaches [14-17]. In particular, contrary to the Gibbons-Hawking prescription [18], it was pointed out that the supergravity Lagrangian should be supersymmetric invariant without imposing Dirichlet boundary conditions. Interestingly, it was recently shown in ref. [19] that the introduction of a supersymmetric extension of the Gauss-Bonnet term in a $\mathcal{N}=1$ and $\mathcal{N}=2$ supergravity Lagrangian (with cosmological constant) allows to recover supersymmetry invariance. This last result, together with the bosonic ones, suggests that the (super)symmetry invariance of the theory requires the addition of topological terms which besides provide the counterterms that regularize the action.

The study of the boundary contributions needed to recover supersymmetry invariance in the presence of matter or bigger supersymmetries remains poorly explored. In this work, 
using a geometrical approach (rheonomic), we explore the boundary terms needed in order to restore a particular enlarged supersymmetry known as AdS-Lorentz.

The AdS-Lorentz (super)algebra is obtained as a deformation of the Maxwell (super)symmetries $[20,21]$, and can be alternatively derived as an abelian semigroup expansion ( $S$-expansion) [22-25] of the AdS (super)algebra [26-29]. As shown in ref. [30,31], it is possible to introduce a generalized cosmological constant term in a Born-Infeld like gravity action when the $A d S$-Lorentz algebra is considered. Analogously, the supersymmetric extension of the AdS-Lorentz algebra allows to introduce a generalized supersymmetric cosmological constant term in a four-dimensional supergravity theory [28].

We shall first present the explicit construction of the bulk Lagrangian in the rheonomic framework. In this geometric approach to supergravity, the duality between a superalgebra and the Maurer-Cartan equations is used to write down the curvatures in the superspace, whose basis is given by the vielbein and the gravitino (bosonic and fermionic directions, respectively). Subsequently, we will study the supersymmetry invariance of the Lagrangian in the presence of a non-trivial boundary. In particular, we will show that the supersymmetric extension of a Gauss-Bonnet like term is required in order to restore the supersymmetry invariance of the full Lagrangian. Interestingly, the supergravity action obtained reproduces a MacDowell-Mansouri type action [32].

\section{$2 \quad A d S$-Lorentz supergravity and rheonomy approach}

In the geometric framework the variational field equations obtained from the Lagrangian are written in terms of exterior differential forms, excluding the Hodge duality operator. Therefore they can be implemented either on the $x$-space manifold, or on any larger manifold containing the $x$-space. In particular, if they are implemented on the full superspace, one obtains algebraic relations between curvature components in $x$-space and curvature components in directions orthogonal to $x$-space. When it happens, the former completely determines the latter, and a solution of the field equations on the $x$-space submanifold can be uniquely extended to a solution of the whole group manifold. The possibility of this lifting is called rheonomy.

This rheonomic lifting can also be viewed as an $x$-space transformation of the fields, which maps solutions of the $x$-space field equations into new solutions. From this point of view, it is nothing other than the on-shell supersymmetry transformation.

The principal demand of any supergravity theory is the invariance of the Lagrangian under supersymmetry transformations. In the rheonomic (geometric) approach, the bosonic one-form $V^{a}(a=0,1,2,3)$ and the fermionic one-form $\psi^{\alpha}(\alpha=1, \ldots, 4)$ define the supervielbein basis in superspace [33]. In this framework, the supersymmetry invariance is satisfied requiring that the Lie derivative of the Lagrangian vanishes for diffeomorphisms in the fermionic directions of superspace,

$$
\delta_{\epsilon} \mathcal{L}=l_{\epsilon} \mathcal{L}=\imath_{\epsilon} d \mathcal{L}+d\left(\imath_{\epsilon} \mathcal{L}\right)=0 .
$$

When a supergravity Lagrangian is considered on space-times without boundary, the condition (2.1) trivially reduces to the first contribution such that $\left.\imath_{\epsilon} \mathcal{L}\right|_{\partial \mathcal{M}}=0$. However, in the presence of a non-trivial boundary the condition (2.1) requires a more subtle treatment. 
Before analyzing $\mathcal{N}=1, D=4$ AdS-Lorentz supergravity in the presence of a nontrivial boundary, we will first study the construction of the bulk Lagrangian and the corresponding supersymmetry transformation laws. First of all, we will apply the rheonomic approach to derive the parametrization of the AdS-Lorentz curvatures by studying the different sectors of the Bianchi Identities.

\subsection{Curvatures parametrization}

The four-dimensional AdS-Lorentz superalgebra is generated by $\left\{J_{a b}, P_{a}, Z_{a b}, Q_{\alpha}\right\}$, whose generators satisfy the (anti)commutation relations

$$
\begin{aligned}
{\left[J_{a b}, J_{c d}\right] } & =\eta_{b c} J_{a d}-\eta_{a c} J_{b d}-\eta_{b d} J_{a c}+\eta_{a d} J_{b c}, \\
{\left[J_{a b}, Z_{c d}\right] } & =\eta_{b c} Z_{a d}-\eta_{a c} Z_{b d}-\eta_{b d} Z_{a c}+\eta_{a d} Z_{b c}, \\
{\left[Z_{a b}, Z_{c d}\right] } & =\eta_{b c} Z_{a d}-\eta_{a c} Z_{b d}-\eta_{b d} Z_{a c}+\eta_{a d} Z_{b c}, \\
{\left[J_{a b}, P_{c}\right] } & =\eta_{b c} P_{a}-\eta_{a c} P_{b}, \quad\left[P_{a}, P_{b}\right]=Z_{a b}, \\
{\left[Z_{a b}, P_{c}\right] } & =\eta_{b c} P_{a}-\eta_{a c} P_{b}, \\
{\left[J_{a b}, Q_{\alpha}\right] } & =-\frac{1}{2}\left(\gamma_{a b} Q\right)_{\alpha}, \quad\left[P_{a}, Q_{\alpha}\right]=-\frac{1}{2}\left(\gamma_{a} Q\right)_{\alpha}, \\
{\left[Z_{a b}, Q_{\alpha}\right] } & =-\frac{1}{2}\left(\gamma_{a b} Q\right)_{\alpha}, \\
\left\{Q_{\alpha}, Q_{\beta}\right\} & =-\frac{1}{2}\left[\left(\gamma^{a b} C\right)_{\alpha \beta} Z_{a b}-2\left(\gamma^{a} C\right)_{\alpha \beta} P_{a}\right] .
\end{aligned}
$$

Here $C$ stands for the charge conjugation matrix and $\gamma_{a}, \gamma_{a b}$ are Dirac matrices. Let us notice that the Lorentz type algebra $\mathcal{L}=\left\{J_{a b}, Z_{a b}\right\}$ is a subalgebra of the above superalgebra. This subalgebra and its extensions to higher dimensions have been useful to derive General Relativity from Born-Infeld gravity theories [34-36]. Further generalizations of the AdSLorentz superalgebra containing more than one spinor charge $Q$ can be found in ref. [28] which can be seen as a deformation of the minimal Maxwell superalgebras [37-40]. Interestingly, the following redefinition of the generators $J_{a b} \rightarrow J_{a b}, Z_{a b} \rightarrow \frac{1}{\bar{e}^{2}} Z_{a b}, P_{a} \rightarrow \frac{1}{\bar{e}} P_{a}$, $Q_{\alpha} \rightarrow \frac{1}{\bar{e}} Q_{\alpha}$ provides us with the non-standard Maxwell superalgebra in the limit $\bar{e} \rightarrow 0$. Let us note that the AdS-Lorentz superalgebra, corresponds to a supersymmetric extension of the $\mathfrak{C}_{4}$ algebra. The $\mathfrak{C}_{m}$ algebras have been of particular interest in order to derive different Lovelock gravity actions from Chern-Simons and Born-Infeld gravity theories [31, 41].

Let us consider the Lorentz type curvatures in the superspace which are given by

$$
\begin{aligned}
\mathcal{R}^{a b} & =d \omega^{a b}+\omega_{c}^{a} \omega^{c b}, \\
R^{a} & =D_{\omega} V^{a}+k_{b}^{a} V^{b}-\frac{1}{2} \bar{\psi} \gamma^{a} \psi, \\
\mathcal{F}^{a b} & =D_{\omega} k^{a b}+k_{c}^{a} k^{c b}, \\
\rho & =D_{\omega} \psi+\frac{1}{4} k^{a b} \gamma_{a b} \psi,
\end{aligned}
$$


where $D_{\omega}=d+\omega$ is the Lorentz covariant exterior derivative. They satisfy the Bianchi identities:

$$
\begin{aligned}
D_{\omega} \mathcal{R}^{a b} & =0, \\
D_{\omega} R^{a} & =\mathcal{R}_{b}^{a} V^{b}+\mathcal{F}_{b}^{a} V^{b}+R^{c} k_{c}^{a}+\bar{\psi} \gamma^{a} \rho, \\
D_{\omega} \mathcal{F}^{a b} & =\mathcal{R}_{c}^{a} k^{c b}-\mathcal{R}_{c}^{b} k^{c a}+\mathcal{F}_{c}^{a} k^{c b}-\mathcal{F}_{c}^{b} k^{c a}, \\
D_{\omega} \rho & =\frac{1}{4} \mathcal{R}_{a b} \gamma^{a b} \psi+\frac{1}{4} \mathcal{F}_{a b} \gamma^{a b} \psi-\frac{1}{4} k_{a b} \gamma^{a b} \rho .
\end{aligned}
$$

The most general Ansatz for the Lorentz type curvatures in the super-vielbein basis $\left(V^{a}, \psi\right)$ of the superspace is given by

$$
\begin{aligned}
\mathcal{R}^{a b} & =\mathcal{R}^{a b}{ }_{c d} V^{c} V^{d}+\bar{\Theta}^{a b}{ }_{c} \psi V^{c}+\alpha \bar{e} \bar{\psi} \gamma^{a b} \psi, \\
R^{a} & =R_{c d}^{a} V^{c} V^{d}+\bar{\Theta}_{c}^{a} \psi V^{c}+\xi \bar{e} \bar{\psi} \gamma^{a} \psi, \\
\mathcal{F}^{a b} & =\mathcal{F}^{a b}{ }_{c d}^{c} V^{c} V^{d}+\bar{\Lambda}_{c}^{a b} \psi V^{c}+\beta \bar{e} \bar{\psi} \gamma^{a b} \psi, \\
\rho & =\rho_{a b} V^{a} V^{b}+\delta \bar{e} \gamma_{a} \psi V^{a}+\Omega_{\alpha \beta} \psi^{\alpha} \psi^{\beta} .
\end{aligned}
$$

where $\bar{e}$ is the rescaling parameter. Setting $R^{a}=0$, we can withdraw some terms appearing in the curvatures, through the study of the scaling constraints. On the other hand, the coefficients $\alpha, \beta, \xi$ and $\delta$ appearing in the Ansatz can be determined considering the parametrization involved in the Bianchi identities in the superspace (2.14)-(2.17) and studying their various sectors. We obtain that the Bianchi identities are satisfied when:

$$
\begin{aligned}
\mathcal{R}^{a b} & =\mathcal{R}^{a b}{ }_{c d} V^{c} V^{d}+\bar{\Theta}^{a b}{ }_{c} \psi V^{c}, \\
R^{a} & =0, \\
\mathcal{F}^{a b} & =\mathcal{F}_{c d}^{a b} V^{c} V^{d}+\bar{\Lambda}_{c}^{a b} \psi V^{c}+\bar{e} \bar{\psi} \gamma^{a b} \psi, \\
\rho & =\rho_{a b} V^{a} V^{b}-\bar{e} \gamma_{a} \psi V^{a},
\end{aligned}
$$

where $\bar{\Theta}_{c}^{a b}=\bar{\Lambda}_{c}^{a b}=\epsilon^{a b d e}\left(\bar{\rho}_{c d} \gamma_{e} \gamma_{5}+\rho_{e c} \gamma_{d} \gamma_{5}-\rho_{d e} \gamma_{c} \gamma_{5}\right)$. In this way we have found the parametrization of the curvatures and we can now consider the rheonomic construction of the bulk Lagrangian in the geometric approach.

\subsection{Rheonomic construction of the Lagrangian}

Following the building rules for the construction of rheonomic Lagrangians [33], we start by writing the most general Ansatz for the Lagrangian as follows

$$
\mathcal{L}=\nu^{(4)}+F^{A} \nu_{A}^{(2)}+F^{A} F^{B} \nu_{A B}^{(0)},
$$

where the super-index $(p)$ denotes a $p$-form and $F^{A}$ are the super $A d S$-Lorentz Lie algebra valued curvatures defined by

$$
\begin{aligned}
\mathcal{R}^{a b} & =d \omega^{a b}+\omega_{c}^{a} \omega^{c b}, \\
R^{a} & =D_{\omega} V^{a}+k_{b}^{a} V^{b}-\frac{1}{2} \bar{\psi} \gamma^{a} \psi, \\
F^{a b} & =D_{\omega} k^{a b}+k_{c}^{a} k^{c b}+4 \bar{e}^{2} V^{a} V^{b}+\bar{e} \psi \gamma^{a b} \psi, \\
\Psi & =D_{\omega} \psi+\frac{1}{4} k^{a b} \gamma_{a b} \psi-\bar{e} \gamma_{a} \psi V^{a},
\end{aligned}
$$


and where

$$
\begin{aligned}
\nu^{(4)}= & \alpha_{1} \epsilon_{a b c d} V^{a} V^{b} V^{c} V^{d}+\alpha_{2} \bar{\psi} \gamma^{a b} \psi V^{c} V^{d} \epsilon_{a b c d}+\alpha_{3} \bar{\psi} \gamma_{a b} \psi V^{a} V^{b} \\
F^{A} \nu_{A}^{(2)}= & \gamma_{1} \epsilon_{a b c d} \mathcal{R}^{a b} V^{c} V^{d}+\gamma_{2} \epsilon_{a b c d} F^{a b} V^{c} V^{d}+\gamma_{3} \bar{\Psi} \gamma_{5} \gamma_{a} \psi V^{a}+\gamma_{4} \bar{\Psi} \gamma_{a} \psi V^{a}+ \\
& \gamma_{5} R^{a} \bar{\psi} \gamma_{a} \psi+\gamma_{6} \mathcal{R}^{a b} \bar{\psi} \gamma_{a b} \psi+\gamma_{7} \mathcal{R}^{a b} V_{a} V_{b}+\gamma_{8} \epsilon_{a b c d} \mathcal{R}^{a b} \bar{\psi} \gamma^{c d} \psi+ \\
& +\gamma_{9} F^{a b} V_{a} V_{b}+\gamma_{10} \epsilon_{a b c d} F^{a b} \bar{\psi} \gamma^{c d} \psi+\gamma_{11} F^{a b} \bar{\psi} \gamma_{a b} \psi \\
F^{A} F^{B} \nu_{A B}^{(0)}= & \beta_{1} \mathcal{R}^{a b} \mathcal{R}_{a b}+\beta_{2} F^{a b} F_{a b}+\beta_{3} \epsilon_{a b c d} \mathcal{R}^{a b} \mathcal{R}^{c d}+\beta_{4} \epsilon_{a b c d} \mathcal{R}^{a b} F^{c d}+ \\
& +\beta_{5} \epsilon_{a b c d} F^{a b} F^{c d}+\beta_{6} \bar{\Psi} \Psi+\beta_{7} \bar{\Psi} \gamma_{5} \Psi+\beta_{8} R^{a} R_{a}
\end{aligned}
$$

with $\alpha_{i}, \beta_{j}, \gamma_{k}$ being constants. Note that the curvatures (2.27)-(2.30) are invariant under the rescaling $\omega^{a b} \rightarrow \omega^{a b}, k^{a b} \rightarrow k^{a b}, V^{a} \rightarrow w V^{a}, \psi \rightarrow w^{1 / 2} \psi$ and $\bar{e} \rightarrow w^{-1} \bar{e}$. Additionally, the Lagrangian must scale with $w^{2}$, being $w^{2}$ the scale-weight of the Einstein term. We can prove that the term $R^{a} R_{a}$ in (2.33) is linear in the curvature. Furthermore, due to scaling constraints reasons, some of the terms in (2.33) disappear. Here we have to observe that a theory in AdS includes a cosmological constant and, since the coefficients appearing in the Lagrangian can be dimensional objects and scale with negative powers of $\bar{e}$, some of the terms in $F^{A} F^{B} \nu_{A B}^{(0)}$ can survive the scaling and contribute to the Lagrangian as total derivatives. However, since we are now constructing the bulk Lagrangian, we can neglect them and set $F^{A} F^{B} \nu_{A B}^{(0)}=0$. We will show that these terms will be fundamental for the construction of the boundary Lagrangian.

Let us consider now the scaling in (2.31) whose coefficients must be redefined in the following way in order to give non-vanishing contributions to the Lagrangian:

$$
\alpha_{1} \equiv \bar{e}^{2} \alpha_{1}^{\prime}, \quad \alpha_{2} \equiv \bar{e} \alpha_{2}^{\prime}, \quad \alpha_{3} \equiv \bar{e} \alpha_{3}^{\prime} .
$$

In this way, all the terms in $\nu$ scale as $w^{2}$. Then, applying the scaling and the parity conservation law to (2.31) and (2.32) we obtain

$$
\alpha_{3}=0 ; \quad \gamma_{4}=\gamma_{5}=\gamma_{6}=\gamma_{7}=\gamma_{8}=\gamma_{9}=\gamma_{10}=\gamma_{11}=0 .
$$

Therefore, we are left with the Lagrangian

$$
\begin{aligned}
\mathcal{L}= & \epsilon_{a b c d} \mathcal{R}^{a b} V^{c} V^{d}+\gamma_{3} \bar{\psi} \gamma_{a} \gamma_{5} \Psi V^{a}+\gamma_{2} \epsilon_{a b c d} F^{a b} V^{c} V^{d} \\
& +\alpha_{1}^{\prime} \bar{e}^{2} \epsilon_{a b c d} V^{a} V^{b} V^{c} V^{d}+\alpha_{2}^{\prime} \bar{e} \epsilon_{a b c d} \bar{\psi} \gamma^{a b} \psi V^{c} V^{d}
\end{aligned}
$$

where we have consistently set $\gamma_{1}=1$. Using the definition of the AdS-Lorentz curvatures $(2.27)-(2.30)$, we can write

$$
\begin{aligned}
\mathcal{L}= & \epsilon_{a b c d} \mathcal{R}^{a b} V^{c} V^{d}+\gamma_{3} \bar{\psi} \gamma_{a} \gamma_{5} D_{\omega} \psi V^{a}+\frac{\gamma_{3}}{4} \epsilon_{a b c d} k^{a b} \bar{\psi} \gamma^{c} \psi V^{d} \\
& +\gamma_{2} \epsilon_{a b c d}\left(D_{\omega} k^{a b}+k_{c}^{a} k^{c b}\right) V^{c} V^{d}+\left(\alpha_{1}^{\prime}+4 \gamma_{2}\right) \bar{e}^{2} \epsilon_{a b c d} V^{a} V^{b} V^{c} V^{d} \\
& +\left(\alpha_{2}^{\prime}+\gamma_{2}+\frac{\gamma_{3}}{2}\right) \bar{e} \epsilon_{a b c d} \bar{\psi} \gamma^{a b} \psi V^{c} V^{d} .
\end{aligned}
$$


We can now determine the coefficients $\alpha_{1}^{\prime}, \alpha_{2}^{\prime}, \gamma_{2}$ and $\gamma_{3}$ through the study of the field equations. In order to obtain them, let us compute the variation of the Lagrangian with respect to the different fields. The variation of the Lagrangian with respect to the spin connection $\omega^{a b}$ is given by

$$
\delta_{\omega} \mathcal{L}=2 \epsilon_{a b c d} \delta \omega^{a b}\left(D_{\omega} V^{c}+\gamma_{2} k_{f}^{c} V^{f}-\frac{1}{8} \gamma_{3} \bar{\psi} \gamma^{c} \psi\right) V^{d} .
$$

Here we see that, if $\gamma_{2}=1$ and $\gamma_{3}=4, \delta_{\omega} \mathcal{L}=0$ leads to the field equation for the AdS-Lorentz supertorsion:

$$
\epsilon_{a b c d} R^{c} V^{d}=0 .
$$

The variation of the Lagrangian with respect to $k^{a b}$ gives the same result.

On the other hand, the variation of the Lagrangian with respect to the vielbein $V^{a}$ leads to

$$
2 \epsilon_{a b c d}\left(\mathcal{R}^{a b} V^{c}+F^{a b} V^{c}\right)+4 \bar{\psi} \gamma_{d} \gamma_{5} \Psi=0
$$

where we have used

$$
\epsilon_{a b c d} k^{a b} \bar{\psi} \gamma^{c} \psi=\bar{\psi} \gamma_{d} \gamma_{5} k^{a b} \gamma_{a b} \psi
$$

and where we have set $\alpha_{1}^{\prime}=-2$ and $\alpha_{2}^{\prime}=-1$, in order to recover the AdS-Lorentz curvatures. In the same way, from the variation with respect to the gravitino field $\psi$ we find the following field equation:

$$
8 V^{a} \gamma_{a} \gamma_{5} \Psi+4 \gamma_{a} \gamma_{5} \psi R^{a}=0 .
$$

Summarizing, we have found the following values for the coefficients:

$$
\alpha_{1}^{\prime}=-2, \quad \alpha_{2}^{\prime}=-1, \quad \gamma_{2}=1, \quad \gamma_{3}=4 .
$$

Thus we have completely determined the bulk Lagrangian $\mathcal{L}_{\text {bulk }}$ of the theory, which can be written in terms of the Lorentz type curvatures (2.10)-(2.13) as follows

$$
\begin{aligned}
\mathcal{L}_{\text {bulk }}= & \epsilon_{a b c d} \mathcal{R}^{a b} V^{c} V^{d}+\epsilon_{a b c d} \mathcal{F}^{a b} V^{c} V^{d}+4 \bar{\psi} \gamma_{a} \gamma_{5} \rho V^{a} \\
& +2 \bar{e}^{2} \epsilon_{a b c d} V^{a} V^{b} V^{c} V^{d}+2 \bar{e} \epsilon_{a b c d} \bar{\psi} \gamma^{a b} \psi V^{c} V^{d} .
\end{aligned}
$$

\subsection{Supersymmetry transformation laws}

The parametrizations we got in the previous section allow to obtain the supersymmetry transformation laws. Indeed, in the rheonomic formalism, the transformations on spacetime are given by

$$
\delta \mu^{A}=(\nabla \epsilon)^{A}+l_{\epsilon} F^{A},
$$

where $\epsilon^{A} \equiv\left(\epsilon^{a b}, \epsilon^{a}, \varepsilon^{a b}, \epsilon\right)$. Then, restricting us to supersymmetric transformations we have $\epsilon^{a b}=\epsilon^{a}=\varepsilon^{a b}=0$ and

$$
\begin{aligned}
l_{\epsilon}\left(\mathcal{R}^{a b}\right) & =\bar{\Theta}^{a b}{ }_{c} \epsilon V^{c}, \\
l_{\epsilon}\left(R^{a}\right) & =0 \\
l_{\epsilon}\left(\mathcal{F}^{a b}\right) & =\bar{\Lambda}^{a b}{ }_{c} \epsilon V^{c}+2 \overline{e \bar{\epsilon}} \gamma^{a b} \psi, \\
l_{\epsilon}(\rho) & =-\bar{e} \gamma_{a} \epsilon V^{a},
\end{aligned}
$$


which provide the following supersymmetry transformation laws:

$$
\begin{aligned}
\delta_{\epsilon} \omega^{a b} & =\bar{\Theta}^{a b}{ }_{c} \epsilon V^{c}, \\
\delta_{\epsilon} V^{a} & =\bar{\epsilon} \gamma^{a} \psi, \\
\delta_{\epsilon} k^{a b} & =-2 \bar{e} \bar{\epsilon} \gamma^{a b} \psi+\bar{\Lambda}^{a b}{ }_{c} \epsilon V^{c}, \\
\delta_{\epsilon} \psi & =d \epsilon+\frac{1}{4} \omega^{a b} \gamma_{a b} \epsilon+\frac{1}{4} k^{a b} \gamma_{a b} \epsilon+\bar{e} \gamma_{a} \epsilon V^{a} .
\end{aligned}
$$

Under these transformation laws the Lagrangian is invariant up to boundary terms. The presence of a boundary requires to check explicitly the condition (2.1).

\section{Supersymmetry invariance in the presence of a boundary}

In this section, following the approach presented in ref. [19], we analyze the supersymmetry invariance of the Lagrangian in the presence of a non-trivial boundary. In particular, we present the explicit boundary terms required in order to recover the full supersymmetry invariance of the Lagrangian.

Let us consider the Lagrangian found in the previous section,

$$
\begin{aligned}
\mathcal{L}_{\text {bulk }}= & \epsilon_{a b c d} \mathcal{R}^{a b} V^{c} V^{d}+4 \bar{\psi} V^{a} \gamma_{a} \gamma_{5} \rho \\
& +\epsilon_{a b c d}\left(\mathcal{F}^{a b} V^{c} V^{d}+2 \bar{e} V^{a} V^{b} \bar{\psi} \gamma^{c d} \psi+2 \bar{e}^{2} V^{a} V^{b} V^{c} V^{d}\right) .
\end{aligned}
$$

The supersymmetry invariance in the bulk is satisfied on-shell

$$
R^{a}=0 .
$$

Nevertheless, the boundary invariance of the Lagrangian under supersymmetry is not trivially satisfied:

$$
\left.l_{\epsilon} \mathcal{L}_{\text {bulk }}\right|_{\partial \mathcal{M}_{4}} \neq 0 .
$$

In order to recover the supersymmetric invariance of the theory, we require a more subtle approach. Indeed, we have to add boundary terms to the bulk Lagrangian.

The only boundary contributions compatible with parity, Lorentz-like invariance and $\mathcal{N}=1$ supersymmetry are

$$
\begin{aligned}
d\left(\varpi^{a b} \mathcal{N}^{c d}+\varpi_{f}^{a} \varpi^{f b} \varpi^{c d}\right) \epsilon_{a b c d} & =\epsilon_{a b c d} \mathcal{N}^{a b} \mathcal{N}^{c d}, \\
d\left(\bar{\rho} \gamma_{5} \psi\right) & =\bar{\rho} \gamma_{5} \rho+\frac{1}{8} \epsilon_{a b c d} \mathcal{N}^{a b} \bar{\psi} \gamma^{c d} \psi,
\end{aligned}
$$

where we have defined $\varpi^{a b}=\omega^{a b}+k^{a b}$ and $\mathcal{N}^{a b}=\mathcal{R}^{a b}+\mathcal{F}^{a b}$, with $\mathcal{R}^{a b}$ and $\mathcal{F}^{a b}$ given by eqs. (2.10) and (2.12), respectively. One can notice that $\varpi^{a b}$ and $\mathcal{N}^{a b}$ are related to a Lorentz-like generator $M_{a b}=J_{a b}+Z_{a b}$ (see eqs. (2.2)-(2.4)). Thus, let us consider the following boundary Lagrangian

$$
\begin{aligned}
\mathcal{L}_{b d y}= & \alpha \epsilon_{a b c d}\left(\mathcal{R}^{a b} \mathcal{R}^{c d}+2 \epsilon_{a b c d} \mathcal{R}^{a b} \mathcal{F}^{c d}+\epsilon_{a b c d} \mathcal{F}^{a b} \mathcal{F}^{c d}\right) \\
& +\beta\left(\bar{\rho} \gamma_{5} \rho+\frac{1}{8} \epsilon_{a b c d} \mathcal{R}^{a b} \bar{\psi} \gamma^{c d} \psi+\frac{1}{8} \epsilon_{a b c d} \mathcal{F}^{a b} \bar{\psi} \gamma^{c d} \psi\right) .
\end{aligned}
$$


Let us note that the structure of a supersymmetric Gauss-Bonnet like gravity appears. Then, the full Lagrangian is given by

$$
\begin{aligned}
\mathcal{L}_{\text {full }}= & \mathcal{L}_{\text {bulk }}+\mathcal{L}_{b d y} \\
= & \epsilon_{a b c d} \mathcal{R}^{a b} V^{c} V^{d}+4 \bar{\psi} V^{a} \gamma_{a} \gamma_{5} \rho+\epsilon_{a b c d}\left(\mathcal{F}^{a b} V^{c} V^{d}+2 \bar{e} V^{a} V^{b} \bar{\psi} \gamma^{c d} \psi+2 \bar{e}^{2} V^{a} V^{b} V^{c} V^{d}\right) \\
& +\alpha \epsilon_{a b c d}\left(\mathcal{R}^{a b} \mathcal{R}^{c d}+2 \epsilon_{a b c d} \mathcal{R}^{a b} \mathcal{F}^{c d}+\epsilon_{a b c d} \mathcal{F}^{a b} \mathcal{F}^{c d}\right) \\
& +\beta\left(\frac{1}{8} \epsilon_{a b c d} \mathcal{R}^{a b} \bar{\psi} \gamma^{c d} \psi+\frac{1}{8} \epsilon_{a b c d} \mathcal{F}^{a b} \bar{\psi} \gamma^{c d} \psi+\bar{\rho} \gamma_{5} \rho\right) .
\end{aligned}
$$

Due to the $\bar{e}^{-2}$-homogeneous scaling of the Lagrangian, we have that the coefficients $\alpha$ and $\beta$ must be related to $\bar{e}^{-2}$ and $\bar{e}^{-1}$, respectively.

As we have previously pointed out, the supersymmetry invariance of the full Lagrangian $\mathcal{L}_{\text {full }}$ requires the following condition

$$
\delta_{\epsilon} \mathcal{L}_{\text {full }}=l_{\epsilon} \mathcal{L}_{\text {full }}=\imath_{\epsilon} d \mathcal{L}_{\text {full }}+d\left(\imath_{\epsilon} \mathcal{L}_{\text {full }}\right)=0
$$

Naturally, the condition for supersymmetry in the bulk $\imath_{\epsilon} d \mathcal{L}_{\text {full }}=0$ is satisfied since the boundary contributions correspond to total derivatives. Thus the supersymmetry invariance of the full Lagrangian $\mathcal{L}_{\text {full }}$ requires to verify the condition $\imath_{\epsilon}\left(\mathcal{L}_{\text {full }}\right)=0$ on the boundary. In particular, we have

$$
\begin{aligned}
\imath_{\epsilon}\left(\mathcal{L}_{\text {full }}\right)= & \epsilon_{a b c d} \imath_{\epsilon}\left(\mathcal{R}^{a b}+\mathcal{F}^{a b}\right) V^{c} V^{d}+4 \bar{\epsilon} V^{a} \gamma_{a} \gamma_{5} \rho+4 \bar{\psi} V^{a} \gamma_{a} \gamma_{5} \imath_{\epsilon}(\rho) \\
& +\epsilon_{a b c d} 4 \bar{e} V^{a} V^{b} \bar{\epsilon} \gamma^{c d} \psi+2 \imath_{\epsilon}\left(\mathcal{R}^{a b}+\mathcal{F}^{a b}\right)\left\{\alpha \mathcal{R}^{c d}+\frac{\beta}{16} \bar{\psi} \gamma^{c d} \psi+\alpha \mathcal{F}^{c d}\right\} \epsilon_{a b c d} \\
& +\frac{\beta}{4} \epsilon_{a b c d}\left(\mathcal{R}^{a b}+\mathcal{F}^{a b}\right) \bar{\epsilon} \gamma^{c d} \psi+2 \beta \imath_{\epsilon}(\bar{\rho}) \gamma_{5} \rho
\end{aligned}
$$

Then, $\left.\frac{\delta \mathcal{L}_{\text {full }}}{\delta \mu^{A}}\right|_{\partial \mathcal{M}}=0$ implies the following constraints on the boundary:

$$
\begin{aligned}
\left.\left(\mathcal{R}^{a b}+\mathcal{F}^{a b}\right)\right|_{\partial \mathcal{M}} & =-\frac{1}{2 \alpha} V^{a} V^{b}-\frac{\beta}{16 \alpha} \bar{\psi} \gamma^{a b} \psi, \\
\left.\rho\right|_{\partial \mathcal{M}} & =\frac{2}{\beta} V^{a} \gamma_{a} \psi .
\end{aligned}
$$

The supersymmetry invariance requires $\left.\imath_{\epsilon}\left(\mathcal{L}_{\text {full }}\right)\right|_{\partial \mathcal{M}}=0$. Thus we find

$$
\begin{aligned}
\left.\imath_{\epsilon}\left(\mathcal{L}_{\text {full }}\right)\right|_{\partial \mathcal{M}}= & -\frac{\beta}{8 \alpha} \epsilon_{a b c d} \bar{\epsilon} \gamma^{a b} \psi V^{c} V^{d}+4 \bar{\epsilon} V^{a} \gamma_{a} \gamma_{5} \rho+\frac{8}{\beta} \bar{\psi} V^{a} \gamma_{a} \gamma_{5} V^{b} \gamma_{b} \epsilon \\
& +4 \bar{e} \epsilon_{a b c d} V^{a} V^{b} \bar{\epsilon} \gamma^{c d} \psi-\left(\frac{\beta}{4 \alpha} \bar{\epsilon} \gamma^{a b} \psi\right)\left\{\alpha \mathcal{R}^{c d}+\frac{\beta}{16} \bar{\psi} \gamma^{c d} \psi+\alpha \mathcal{F}^{c d}\right\} \epsilon_{a b c d} \\
& +\frac{\beta}{4} \epsilon_{a b c d}\left\{\mathcal{R}^{a b} \bar{\epsilon} \gamma^{c d} \psi+\mathcal{F}^{a b} \bar{\epsilon} \gamma^{c d} \psi\right\}-4 \bar{\epsilon} \gamma_{a} V^{a} \gamma_{5} \rho .
\end{aligned}
$$

Using the Fierz identity for $\mathcal{N}=1, \gamma_{a b} \psi \bar{\psi} \gamma^{a b} \psi=0$, we have

$$
\left.\imath_{\epsilon}\left(\mathcal{L}_{\text {full }}\right)\right|_{\partial \mathcal{M}}=\left(4 \bar{e}-\frac{\beta}{8 \alpha}\right) \epsilon_{a b c d} \bar{\epsilon} \gamma^{a b} \psi V^{c} V^{d}+\frac{8}{\beta} \bar{\psi} V^{a} \gamma_{a} \gamma_{5} V^{b} \gamma_{b} \epsilon
$$


Then, using the gamma matrices identity, we have that the supersymmetry invariance implies the following relation between $\alpha$ and $\beta$ :

$$
\frac{\beta}{4 \alpha}+\frac{8}{\beta}=8 \bar{e} .
$$

Solving for $\beta$ we find

$$
\beta=16 e \alpha\left(1 \pm \sqrt{1-\frac{1}{8 \bar{e}^{2} \alpha}}\right) .
$$

Let us note that the root vanishes for

$$
\alpha=\frac{1}{8 \bar{e}^{2}},
$$

which implies

$$
\beta=\frac{2}{\bar{e}} .
$$

Interestingly, with these values for $\alpha$ and $\beta$ we recover the following 2 -form curvatures

$$
\begin{aligned}
N^{a b} & =\mathcal{R}^{a b}+\mathcal{F}^{a b}+4 \bar{e}^{2} V^{a} V^{b}+\bar{e} \bar{\psi} \gamma^{a b} \psi, \\
\Psi & =\rho-\bar{e} V^{a} \gamma_{a} \psi, \\
R^{a} & =D_{\omega} V^{a}+k_{b}^{a} V^{b}-\frac{1}{2} \bar{\psi} \gamma^{a} \psi .
\end{aligned}
$$

which reproduce the AdS-Lorentz curvatures with

$$
\begin{aligned}
& N^{a b}=\mathcal{R}^{a b}+F^{a b}, \quad \text { where } \\
& \mathcal{R}^{a b}=d \omega^{a b}+\omega^{a}{ }_{c} \omega^{c b}, \\
& F^{a b}=\mathcal{F}^{a b}+4 \bar{e}^{2} V^{a} V^{b}+\bar{e} \bar{\psi} \gamma^{a b} \psi .
\end{aligned}
$$

Finally, the full Lagrangian can be written as a MacDowell-Mansouri like form in terms of the 2-form curvatures (3.11)-(3.12),

$$
\mathcal{L}_{\text {full }}=\frac{1}{8 \bar{e}^{2}} \epsilon_{a b c d} N^{a b} N^{c d}+\frac{2}{\bar{e}} \bar{\Psi} \gamma_{5} \Psi,
$$

whose boundary term corresponds to a supersymmetric Gauss-Bonnet like term,

$$
\begin{aligned}
\mathcal{L}_{b d y}= & \frac{1}{8 \bar{e}^{2}} \epsilon_{a b c d}\left(\mathcal{R}^{a b} \mathcal{R}^{c d}+2 \mathcal{R}^{a b} \mathcal{F}^{c d}+\mathcal{F}^{a b} \mathcal{F}^{c d}\right) \\
& +\frac{4}{\bar{e}}\left(\frac{1}{8} \epsilon_{a b c d} \mathcal{R}^{a b} \bar{\psi} \gamma^{c d} \psi+\frac{1}{8} \epsilon_{a b c d} \mathcal{F}^{a b} \bar{\psi} \gamma^{c d} \psi+\bar{\rho} \gamma_{5} \rho\right) .
\end{aligned}
$$

This term allows to recover the supersymmetric invariance of the theory in the presence of a boundary. The same phenomenon occurs in pure gravity, where the Gauss-Bonnet term assures the invariance of the Lagrangian in the presence of a non-trivial boundary. Additionally, the supersymmetric extension of the Gauss-Bonnet term was introduced in ref. [19], in order to restore the supersymmetry invariance in $\mathcal{N}=1$ and $\mathcal{N}=2, O \operatorname{sp}(\mathcal{N} \mid 4)$ supergravity in the presence of a boundary. 
On the other hand, the bulk Lagrangian reproduces the generalized supersymmetric cosmological term presented in ref. [28], and corresponds to a supersymmetric extension of the results found in refs. [30, 42].

Let us note that an Inönü-Wigner (IW) contraction of the full Lagrangian (3.14) leads to the Maxwell MacDowell-Mansouri Lagrangian presented in ref. [43], corresponding to $\mathcal{N}=1$ pure supergravity Lagrangian in the presence of a non-trivial boundary.

\section{Comments and possible developments}

In this paper we have first of all presented the explicit construction of the $\mathcal{N}=1, D=4$ AdS-Lorentz supergravity bulk Lagragian in the rheonomic framework. In particular, we have shown an alternative way to introduce a generalized supersymmetric cosmological term to supergravity. Subsequently, we have studied the supersymmetry invariance of the Lagrangian in the presence of a non-trivial boundary. Interestingly, the supersymmetric extension of a Gauss-Bonnet like term is required in order to restore the supersymmetry invariance of the full Lagrangian. The addition of a topological boundary term in a four-dimensional bosonic action is equivalent to the holographic renormalization in the $A d S / C F T$ formalism. Then, it seems that the presence of the $k^{a b}$ fields through the $\mathcal{F}^{a b}$ curvature in the boundary would allow to regularize the supergravity action in the holographic renormalization language. Additionally, as was pointed out in refs. [44, 45], the bosonic MacDowell-Mansouri action is on-shell equivalent to the square of the Weyl tensor describing conformal gravity. Thus, the supergravity action à la MacDowell-Mansouri would suggest a superconformal structure which represents an additional motivation in our approach.

The results obtained here could be useful in order to study supergravity theories in the presence of a non-trivial boundary in higher dimensions or coupled to matter. In particular, it would be interesting to analyze the boundary terms necessary to restore the supersymmetry invariance of a general matter coupled $\mathcal{N}=2$ supergravity considering the bulk Lagrangians introduced in refs. [46, 47].

\section{Acknowledgments}

This work was supported in part by FONDECYT Grants No 1130653 (MCI) and also by the Newton-Picarte CONICYT Grant No. DPI20140053 (P.K.C. and E.K.R.). MCI was supported by grants from CONICYT and from the Universidad de Concepción, Chile. The authors wish to thank L. Andrianopoli, R. D'Auria and M. Trigiante for enlightening discussions.

Open Access. This article is distributed under the terms of the Creative Commons Attribution License (CC-BY 4.0), which permits any use, distribution and reproduction in any medium, provided the original author(s) and source are credited. 


\section{References}

[1] J.M. Maldacena, The large- $N$ limit of superconformal field theories and supergravity, Int. J. Theor. Phys. 38 (1999) 1113 [hep-th/9711200] [INSPIRE].

[2] S.S. Gubser, I.R. Klebanov and A.M. Polyakov, Gauge theory correlators from noncritical string theory, Phys. Lett. B 428 (1998) 105 [hep-th/9802109] [InSPIRE].

[3] E. Witten, Anti-de Sitter space and holography, Adv. Theor. Math. Phys. 2 (1998) 253 [hep-th/9802150] [INSPIRE].

[4] O. Aharony, S.S. Gubser, J.M. Maldacena, H. Ooguri and Y. Oz, Large-N field theories, string theory and gravity, Phys. Rept. 323 (2000) 183 [hep-th/9905111] [InSPIRE].

[5] V. Balasubramanian and P. Kraus, A stress tensor for Anti-de Sitter gravity, Commun. Math. Phys. 208 (1999) 413 [hep-th/9902121] [InSPIRE].

[6] K. Skenderis, Lecture notes on holographic renormalization, Class. Quant. Grav. 19 (2002) 5849 [hep-th/0209067] [INSPIRE].

[7] M. Bianchi, D.Z. Freedman and K. Skenderis, Holographic renormalization, Nucl. Phys. B 631 (2002) 159 [hep-th/0112119] [INSPIRE].

[8] R. Aros, M. Contreras, R. Olea, R. Troncoso and J. Zanelli, Conserved charges for gravity with locally AdS asymptotics, Phys. Rev. Lett. 84 (2000) 1647 [gr-qc/9909015] [INSPIRE].

[9] R. Aros, M. Contreras, R. Olea, R. Troncoso and J. Zanelli, Conserved charges for even dimensional asymptotically AdS gravity theories, Phys. Rev. D 62 (2000) 044002 [hep-th/9912045] [INSPIRE].

[10] P. Mora, R. Olea, R. Troncoso and J. Zanelli, Finite action principle for Chern-Simons AdS gravity, JHEP 06 (2004) 036 [hep-th/0405267] [INSPIRE].

[11] R. Olea, Mass, angular momentum and thermodynamics in four-dimensional Kerr-AdS black holes, JHEP 06 (2005) 023 [hep-th/0504233] [INSPIRE].

[12] D.P. Jatkar, G. Kofinas, O. Mišković and R. Olea, Conformal mass in AdS gravity, Phys. Rev. D 89 (2014) 124010 [arXiv:1404.1411] [InSPIRE].

[13] D.P. Jatkar, G. Kofinas, O. Mišković and R. Olea, Conformal mass in Einstein-Gauss-Bonnet AdS gravity, Phys. Rev. D 91 (2015) 105030 [arXiv:1501.06861] [INSPIRE].

[14] P. van Nieuwenhuizen and D.V. Vassilevich, Consistent boundary conditions for supergravity, Class. Quant. Grav. 22 (2005) 5029 [hep-th/0507172] [INSPIRE].

[15] D.V. Belyaev, Boundary conditions in supergravity on a manifold with boundary, JHEP 01 (2006) 047 [hep-th/0509172] [INSPIRE].

[16] D.V. Belyaev and P. van Nieuwenhuizen, Tensor calculus for supergravity on a manifold with boundary, JHEP 02 (2008) 047 [arXiv:0711.2272] [INSPIRE].

[17] D.V. Belyaev and P. van Nieuwenhuizen, Simple D $=4$ supergravity with a boundary, JHEP 09 (2008) 069 [arXiv:0806.4723] [INSPIRE].

[18] G.W. Gibbons and S.W. Hawking, Action Integrals and Partition Functions in Quantum Gravity, Phys. Rev. D 15 (1977) 2752.

[19] L. Andrianopoli and R. D'Auria, $N=1$ and $N=2$ pure supergravities on a manifold with boundary, JHEP 08 (2014) 012 [arXiv:1405.2010] [INSPIRE]. 
[20] D.V. Soroka and V.A. Soroka, Semi-simple extension of the (super)Poincaré algebra, Adv. High Energy Phys. 2009 (2009) 234147 [hep-th/0605251] [INSPIRE].

[21] R. Durka, J. Kowalski-Glikman and M. Szczachor, Gauged AdS-Maxwell algebra and gravity, Mod. Phys. Lett. A 26 (2011) 2689 [arXiv:1107.4728] [InSPIRE].

[22] F. Izaurieta, E. Rodríguez and P. Salgado, Expanding Lie (super)algebras through Abelian semigroups, J. Math. Phys. 47 (2006) 123512 [hep-th/0606215] [INSPIRE].

[23] R. Caroca, I. Kondrashuk, N. Merino and F. Nadal, Bianchi spaces and their three-dimensional isometries as $S$-expansions of two-dimensional isometries, J. Phys. A 46 (2013) 225201 [arXiv:1104.3541] [InSPIRE].

[24] L. Andrianopoli, N. Merino, F. Nadal and M. Trigiante, General properties of the expansion methods of Lie algebras, J. Phys. A 46 (2013) 365204 [arXiv:1308.4832] [InSPIRE].

[25] M. Artebani, R. Caroca, M.C. Ipinza, D.M. Peñafiel and P. Salgado, Geometrical aspects of the Lie Algebra S-Expansion Procedure, J. Math. Phys. 57 (2016) 023516 [arXiv: 1602.04525].

[26] J. Diaz et al., A generalized action for $(2+1)$-dimensional Chern-Simons gravity, J. Phys. A 45 (2012) 255207 [arXiv:1311.2215] [inSPIRE].

[27] O. Fierro, F. Izaurieta, P. Salgado and O. Valdivia, $(2+1)$-dimensional supergravity invariant under the AdS-Lorentz superalgebra, arXiv:1401.3697 [INSPIRE].

[28] P.K. Concha, E.K. Rodríguez and P. Salgado, Generalized supersymmetric cosmological term in $N=1$ Supergravity, JHEP 08 (2015) 009 [arXiv:1504.01898] [INSPIRE].

[29] P.K. Concha, R. Durka, N. Merino and E.K. Rodríguez, New family of Maxwell like algebras, Phys. Lett. B 759 (2016) 507 [arXiv:1601.06443] [INSPIRE].

[30] P. Salgado and S. Salgado, $\mathfrak{s o}(D-1,1) \otimes \mathfrak{s o}(D-1,2)$ algebras and gravity, Phys. Lett. B 728 (2014) 5 [INSPIRE].

[31] P.K. Concha, N. Merino and E.K. Rodríguez, Lovelock gravities from Born-Infeld gravity theory, arXiv:1606.07083 [INSPIRE].

[32] S.W. MacDowell and F. Mansouri, Unified Geometric Theory of Gravity and Supergravity, Phys. Rev. Lett. 38 (1977) 739.

[33] L. Castellani, R. D'Auria and P. Fre, Supergravity and superstrings: A geometric perspective. Vol. 1 and 2, World Scientific, Singapore (1991).

[34] P.K. Concha, D.M. Peñafiel, E.K. Rodríguez and P. Salgado, Even-dimensional General Relativity from Born-Infeld gravity, Phys. Lett. B 725 (2013) 419 [arXiv:1309.0062] [INSPIRE].

[35] P.K. Concha, D.M. Peñafiel, E.K. Rodríguez and P. Salgado, Chern-Simons and Born-Infeld gravity theories and Maxwell algebras type, Eur. Phys. J. C 74 (2014) 2741 [arXiv: 1402.0023] [INSPIRE].

[36] P.K. Concha, D.M. Peñafiel, E.K. Rodríguez and P. Salgado, Generalized Poincaré algebras and Lovelock-Cartan gravity theory, Phys. Lett. B 742 (2015) 310 [arXiv:1405.7078] [INSPIRE].

[37] S. Bonanos, J. Gomis, K. Kamimura and J. Lukierski, Maxwell Superalgebra and Superparticle in Constant Gauge Badkgrounds, Phys. Rev. Lett. 104 (2010) 090401 [arXiv:0911.5072] [INSPIRE]. 
[38] J.A. de Azcarraga, J.M. Izquierdo, J. Lukierski and M. Woronowicz, Generalizations of Maxwell (super)algebras by the expansion method, Nucl. Phys. B 869 (2013) 303 [arXiv: 1210.1117] [INSPIRE].

[39] P.K. Concha and E.K. Rodríguez, Maxwell Superalgebras and Abelian Semigroup Expansion, Nucl. Phys. B 886 (2014) 1128 [arXiv: 1405.1334] [InSPIRE].

[40] P.K. Concha, O. Fierro, E.K. Rodríguez and P. Salgado, Chern-Simons supergravity in D $=3$ and Maxwell superalgebra, Phys. Lett. B 750 (2015) 117 [arXiv:1507.02335] [INSPIRE].

[41] P.K. Concha, R. Durka, C. Inostroza, N. Merino and E.K. Rodríguez, Pure Lovelock gravity and Chern-Simons theory, Phys. Rev. D 94 (2016) 024055 [arXiv:1603.09424] [INSPIRE].

[42] J.A. de Azcarraga, K. Kamimura and J. Lukierski, Generalized cosmological term from Maxwell symmetries, Phys. Rev. D 83 (2011) 124036 [arXiv: 1012.4402] [INSPIRE].

[43] P.K. Concha and E.K. Rodríguez, $N=1$ supergravity and Maxwell superalgebras, JHEP 09 (2014) 090 [arXiv:1407.4635] [INSPIRE].

[44] O. Mišković and R. Olea, Topological regularization and self-duality in four-dimensional anti-de Sitter gravity, Phys. Rev. D 79 (2009) 124020 [arXiv:0902.2082] [INSPIRE].

[45] O. Mišković, R. Olea and M. Tsoukalas, Renormalized AdS action and Critical Gravity, JHEP 08 (2014) 108 [arXiv: 1404.5993] [INSPIRE].

[46] L. Andrianopoli et al., $N=2$ supergravity and $N=2$ super Yang-Mills theory on general scalar manifolds: Symplectic covariance, gaugings and the momentum map, J. Geom. Phys. 23 (1997) 111 [hep-th/9605032] [INSPIRE].

[47] L. Andrianopoli, P. Concha, R. D'Auria, E. Rodríguez and M. Trigiante, Observations on BI from $\mathcal{N}=2$ Supergravity and the General Ward Identity, JHEP 11 (2015) 061 [arXiv: 1508.01474] [INSPIRE]. 